\title{
Scattering features and variability of the Crab pulsar
}

\section{Document Version}

Accepted author manuscript

Link to publication record in Manchester Research Explorer

\section{Citation for published version (APA):}

Driessen, L. N., Janssen, G., Bassa, C., Stappers, B., \& Stinebring, D. R. (Accepted/In press). Scattering features and variability of the Crab pulsar.

\section{Citing this paper}

Please note that where the full-text provided on Manchester Research Explorer is the Author Accepted Manuscript or Proof version this may differ from the final Published version. If citing, it is advised that you check and use the publisher's definitive version.

\section{General rights}

Copyright and moral rights for the publications made accessible in the Research Explorer are retained by the authors and/or other copyright owners and it is a condition of accessing publications that users recognise and abide by the legal requirements associated with these rights.

\section{Takedown policy}

If you believe that this document breaches copyright please refer to the University of Manchester's Takedown Procedures [http://man.ac.uk/04Y6Bo] or contact uml.scholarlycommunications@manchester.ac.uk providing relevant details, so we can investigate your claim.

\section{OPEN ACCESS}




\title{
Scattering features and variability of the Crab pulsar
}

\author{
L. N. Driessen ${ }^{1,2,3 \star}$, G. H. Janssen ${ }^{1,4}$, C. G. Bassa ${ }^{1}$, B. W. Stappers ${ }^{3}$, and D. R. Stinebring 5 \\ ${ }^{1}$ ASTRON, the Netherlands Institute for Radio Astronomy, Oude Hoogeveensedijk 4, 7991 PD, Dwingeloo, The Netherlands \\ ${ }^{2}$ Anton Pannekoek Institute for Astronomy, University of Amsterdam, Science Park 904, 1098 XH Amsterdam, The Netherlands \\ ${ }^{3}$ Jodrell Bank Centre for Astrophysics, School of Physics and Astronomy, The University of Manchester, Manchester, M13 9PL, UK \\ ${ }^{4}$ Department of Astrophysics/IMAPP, Radboud University, P.O. Box 9010, 6500 GL Nijmegen, The Netherlands \\ ${ }^{5}$ Department of Physics and Astronomy, Oberlin College, 110 No. Professor St., Oberlin OH 44074, United States
}

Accepted XXX. Received YYY; in original form ZZZ

\begin{abstract}
We report on Westerbork Synthesis Radio Telescope observations of the Crab pulsar at $350 \mathrm{MHz}$ from 2012 November 24 until 2015 June 21. During this period we consistently observe variations in the pulse profile of the Crab. Both variations in the scattering width of the pulse profile as well as delayed copies, also known as echoes, are seen regularly. These observations support the classification of two types of echoes: those that follow the truncated exponential shape expected for the thin-screen scattering approximation, and echoes that show a smoother, more Gaussian shape. During a sequence of high-cadence observations in 2015, we find that these non-exponential echoes evolve in time by approaching the main pulse and interpulse in phase, overlapping the main pulse and interpulse, and later receding. We find a pulse scatter-broadening time scale, $\tau$, scaling with frequency as $v^{\alpha}$, with $\alpha=-3.9 \pm 0.5$, which is consistent with expected values for thin-screen scattering models.
\end{abstract}

Key words: pulsars: PSR B0531+21 - scattering - supernovae: M1

\section{INTRODUCTION}

Pulsar B0531+21 is located within the Crab nebula (M1) and is commonly referred to as the Crab pulsar. The Crab pulsar was formed in a core-collapse supernova which was observed by astronomers in $1054 \mathrm{AD}$. The pulsar itself was discovered in 1968 (Staelin \& Reifenstein 1968). The Crab pulsar is visible at all observable wavelengths and is one of the brightest objects in the radio sky (e.g. Karuppusamy et al. 2010). It has a rotation period of $33.7 \mathrm{~ms}$ and is $2 \mathrm{kpc}$ away (Kaplan et al. 2008). The pulse profile of the Crab pulsar has three main components, which are shown in Fig. 1a: the main pulse (MP) with a precursor, and the interpulse (IP) at $\mathrm{a} \sim 145^{\circ}$ phase offset from the peak of the MP. The Crab pulse profile is known to vary intrinsically over frequency (e.g. Moffett \& Hankins 1996, and references therein).

The Crab pulsar is known to show variability in pulse scatter-broadening, which is seen as broadening of the trailing parts of the profile. Apart from general variability in pulse scatter-broadening, in 1974 (Lyne \& Thorne 1975), 1997 (Backer et al. 2000; Lyne et al. 2001), and 2006 (Kuzmin et al. 2008), anomalous increases in pulse scatter-broadening of the Crab pulsar were observed. During the 1974 and 1997 periods of additional pulse scatter-

^ Contact e-mail: Laura@Driessen.net.au (LND) broadening, a new feature was observed as an extra peak on the trailing edge of both the MP and IP. In these papers, extra features were labelled as "ghosts" or "echoes". An echo was not seen on the precursor as it has a much lower flux density compared to the MP and any extra feature would blend into the MP (Backer et al. 2000). During these periods of increased pulse scatter-broadening an echo would appear as a delayed copy of the pulse profile and over time the delay would decrease until the echo and main profile overlap, after which the delay would increase again. It was observed in 1974 and 1997 that while the echo persisted, the peak flux density of the pulse profile decreased, likely due to the effect of pulse scatter-broadening in combination with an increase in dispersion measure (Graham-Smith et al. 2011).

Generally the pulse scatter-broadening that is seen on the Crab is attributed to the interstellar medium (ISM), while the echo feature is attributed to a second scattering screen (e.g. Backer et al. 2000) or lens (Graham-Smith et al. 2011) close to the Crab pulsar. The Crab pulsar is surrounded by the Crab nebula, which is a plerionic supernova remnant consisting of a pulsar wind nebula surrounded by cold ejecta dust, without the forward and reverse shock typical of young supernova remnants (Owen \& Barlow 2015). It is therefore logical to attribute this extra scattering screen to material in the nebula (e.g. Kuzmin et al. 2008).

Pulse scatter-broadening is a multi-path propagation effect caused by inhomogeneity in electron density between a 


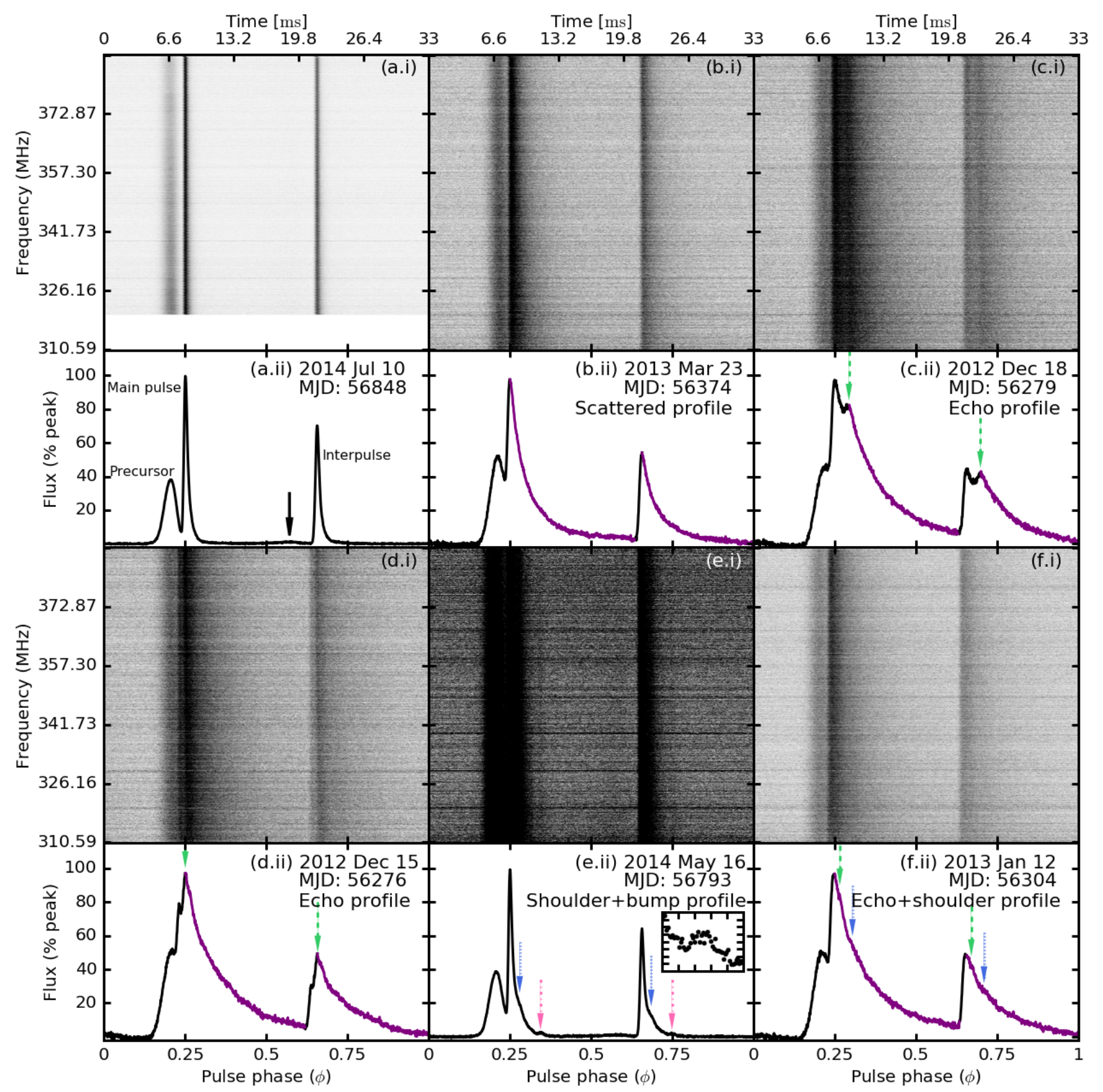

Figure 1. WSRT observations of the Crab pulsar at 350 MHz. Each grey scale plot, showing intensity as a function of frequency and pulse phase, corresponds to the frequency and time averaged pulse profile below. Green (dashed) arrows indicate echo features (c, d and f), blue (dotted) arrows indicate shoulder features (e and f), and pink (dot-dashed) arrows indicate bump features (e). In the grey scale plots the grey scale indicates how bright the features are, with all of the observations scaled linearly such that the maximum is 1.0 (black) and the minimum is 0.0 (white). In plot (e) the grey scale has been adjusted to a minimum of 0.0 and maximum of 0.2 in order to show the shoulder and bump features clearly. In (a) the intrinsic features of the pulse profile of the Crab pulsar at $350 \mathrm{MHz}$ are labelled, including the low-frequency precursor to the IP (Moffett \& Hankins 1996; Karuppusamy et al. 2012) which is indicated with a solid black arrow. (b) shows an example of an observation with additional pulse scatter-broadening (purple). (c) shows an example of an observation with a clear echo feature. In (d) the echo is brighter than the main profile. Plot (e) shows observations with (blue/dashed) shoulder and (pink/dot-dashed) bump features, the inset figure in (e.ii) shows a zoom in on the bump trailing the MP. Plot (f) shows an observation with significant pulse scatter-broadening, an echo (close in phase to the main profile), and a shoulder. 
pulsar and the observer (e.g. Rickett 1969; Rankin et al. 1970; Cordes 1986). The change in path length of light due to material with irregular densities causes a delay in the arrival time of the light from the pulsar. The effect of scattering on pulse profiles is more pronounced at lower frequencies as pulse scatter-broadening time, $\tau$, is related to frequency, $v$, by $\tau \propto v^{\alpha}$. There are several models that are generally used to describe the effects of scattering on a pulsed signal. A thin-screen scattering model is an approximation that assumes that the material causing the pulse scatter-broadening is in a thin screen between the observer and the object (Williamson 1972). The screen is usually attributed to the ISM and it is expected that $-4.4<\alpha<-4$ (Lewandowski et al. 2015); where $\alpha=-4.4$ assumes a Kolmogorov spectrum (Kuzmin et al. 2002) and $\alpha=-4$ assumes Gaussian inhomogeneities (Lang 1971; Lee \& Jokipii 1976), however many pulsars have been observed to have $\alpha$ values greater than -4 and less than -4.4 (Krishnakumar et al. 2017). The frequency dependence of pulse scatter-broadening is shown in Fig. 2. In a pulse profile, the effect of thin-screen scattering appears as a truncated exponential or "exponential tail", which is a broadening of the trailing edge (or "tail") of the profile components (e.g. Lyne \& Thorne 1975; Backer et al. 2000).

In this paper we present and analyse additional pulse scatter-broadening and variable features in the pulse profile of the Crab pulsar in Westerbork Synthesis Radio Telescope (WSRT; Baars \& Hooghoudt 1974) observations at $350 \mathrm{MHz}$. In Sec. 2 we describe our observations and our method for modelling the pulse profiles. In Sec 3 we present our results and in Sec. 4 we discuss the features we see in the pulse profiles. We conclude in Sec. 5 .

\section{OBSERVATIONS AND ANALYSIS}

\subsection{Observations}

We have obtained observations of the Crab pulsar with the WSRT at observing frequencies between 310 and $380 \mathrm{MHz}$ with the PuMaII pulsar instrument (Karuppusamy et al. 2008). PuMa II records real valued, Nyquist sampled timeseries for dual polarizations for 8 subbands of $10 \mathrm{MHz}$ bandwidth each. The subbands were overlapped by $1.25 \mathrm{MHz}$ to mitigate a roll-off of the bandpass, resulting in a total effective bandwidth of $70 \mathrm{MHz}$. The Nyquist sampled timeseries were coherently dedispersed to a $\mathrm{DM}^{1}$ of $56.79 \mathrm{pc} \mathrm{cm}^{-3}$ and folded with the dspsr ${ }^{2}$ (van Straten \& Bailes 2011) software package, resulting in $156.25 \mathrm{kHz}$ wide channels and 1024 pulse phase bins across the profile. A total of 89 observations with integration times varying from 20 to $60 \mathrm{~min}$ were taken between 2012 November 24 and 2015 June 21. We excluded 5 observations that were affected by high sky temperatures due to the Sun passing near the line of sight towards the Crab pulsar (each year around June 15). Due

\footnotetext{
1 Dispersion measure (DM) is a measure of the column density of free electrons between a pulsar and the observer. DM causes a delay in the pulse arrival time, which is more pronounced at lower frequencies. The time delay is related to frequency by $t \propto v^{-2}$.

2 http://dspsr.sourceforge.net
}

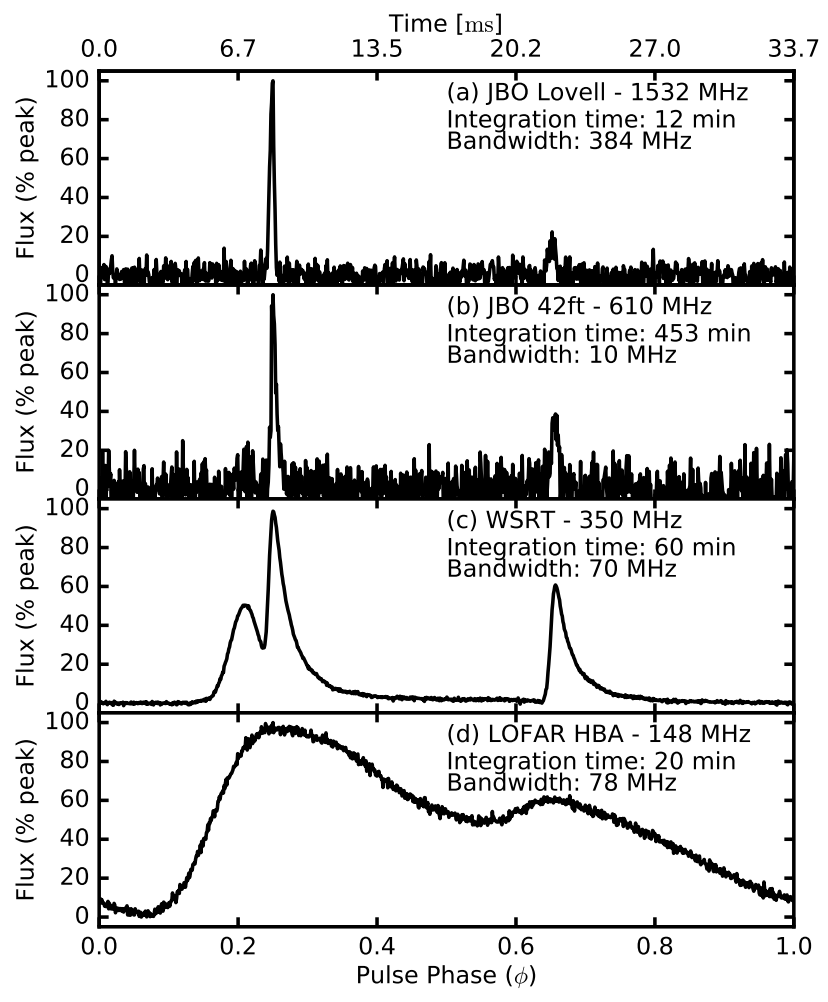

Figure 2. Observations of the Crab pulsar from (a) the Lovell telescope at the Jodrell Bank Observatory (JBO), (b) the $42 \mathrm{ft}$ telescope at JBO, (c) the WSRT, and (d) the LOw Frequency ARray (LOFAR) High Band Antennas (HBA). The Lovell observation was taken on 2015 June 10 , the $42 \mathrm{ft}$ and WSRT observations were both taken on 2015 June 11, the LOFAR observation was taken on 2015 June 12. The effect of pulse scatter-broadening increases as the observing frequency decreases. Apart from the effects of pulse scatter-broadening, the profile evolution with frequency can be seen with the changes in the precursor to the MP. For illustration purposes, the profiles have been scaled such that the peak of the MP is defined at $100 \%$, and the lowest part of the profile is at zero. The intrinsic flux densities are different for each observing frequency. Note that for both the WSRT and LOFAR profiles the baseline is increased due to the pulse scatterbroadening time scale covering significant fractions of the pulse period. The profiles have also been aligned such that the MP is at a pulse phase of 0.25 .

to the interferometric nature of the WSRT array, very little radio frequency interference (RFI) was present in the folded data. Remaining narrow-band RFI was flagged manually using tools from the psrchive ${ }^{3}$ software suite (Hotan et al. 2004). As the DM towards the Crab pulsar varies with time (e.g. Rankin \& Counselman 1973; Kuzmin et al. 2008), we corrected the DM of each observation to the value that minimized the width of the profile components. 


\subsection{Pulse profile features}

The observations of the Crab pulsar show significant variations in the pulse scatter-broadening properties in the $350 \mathrm{MHz}$ observing band. A set of representative observations are shown in Fig. 1 as time averaged and frequency collapsed profiles, and as profiles across a range of frequencies. The time averaged and frequency collapsed profiles for all 84 observations are shown in Appendix A. In observations with low pulse scatter-broadening, such as those in Fig. 1a and Fig. 1e, the precursor to the MP is almost completely separated from the MP, while in observations with high pulse scatter-broadening the two components merge. Low pulse scatter-broadening observations also show the presence of the low-frequency precursor to the IP (indicated by a black arrow in Fig. 1a, which has previously been seen at observing frequencies predominantly below $200 \mathrm{MHz}$ (e.g. Karuppusamy et al. 2012, and references therein) and weakly at $330 \mathrm{MHz}$ (Moffett \& Hankins 1996).

Several observations show delayed copies, or echoes, of the pulse profile on the trailing edge of both the MP and IP. These copies are complete copies of the pulse profile; there is always an echo on the IP if there is one on the MP and vice versa, and there can be one echo, multiple echoes, or no echoes in an observation. Some echoes, such as the echo shown in Fig. 1c, have a sharp rising edge and scatter-broadened trailing edge; we will call these exponential echoes. Other echoes, such as those in Fig. 1e, have small amplitudes, lack a sharp rising edge, and have a more Gaussian-like shape; we will call these non-exponential echoes. For exponential echoes the echo of the precursor is not seen as it overlaps the MP and for non-exponential echoes the amplitude of the precursor echo is not seen as it is below the noise. For clarity, we have distinguished between two types of non-exponential echo, which we will call "shoulders" and "bumps", examples of which are shown in Fig. 1e and f. We will use the term "echo" to refer to the exponential echoes. Fig. 3 shows the epochs where we observe echoes, shoulders, and bumps in our WSRT observations.

\subsection{Pulse profile modelling}

To model the observed pulse profiles and constrain pulse scatter-broadening properties, we implicitly assume that the pulse scatter-broadening can be modelled with the thinscreen scattering approximation. Hence, we model the timedependent pulse scatter-broadening function as a truncated exponential of the form $s(t)=\frac{1}{\tau} \exp \left(-\frac{t}{\tau}\right) H(t)$ (e.g. McKinnon 2014), where $\tau$ is the pulse scatter-broadening time scale, and $H(t)$ the Heaviside step function as a function of time $t$.

The components of the intrinsic pulse profile of the Crab pulsar are modelled with von Mises functions (circular normal distributions $), f(\phi)=A \exp \left(\kappa\left[\cos 2 \pi\left(\phi-\phi_{0}\right)\right]\right)$ where $\phi$ is pulse phase, for a component at pulse phase $\phi_{0}$, amplitude $A$, and reciprocal width $\kappa$. The reciprocal width is related to the Gaussian width $\sigma$ through $\kappa^{-1} \approx \sigma^{2}$. The intrinsic profile is modelled as the sum of three von Mises functions, fitting the pulse phase, amplitude, and reciprocal of the width of the MP, IP, and precursor.

As the pulse scatter-broadening time scale $\tau$ can exceed the spin period $(P=33.7 \mathrm{~ms})$ of the Crab pulsar (e.g. Fig. 1c), we have to ensure that the scattered flux located outside the pulse phase range $0<\phi<1$ is taken into account. To achieve this, the intrinsic profile is defined for one rotation and padded with zeros for several preceding and following rotations. The number of rotations depends on the pulse scatter-broadening time scale $\tau_{\phi}$ (defined in pulse phase as $\left.\tau_{\phi}=\tau / P\right)$, such that the pulse scatter-broadening function at large $t$ becomes negligible. The zero padded intrinsic profile was subsequently convolved with the pulse scatterbroadening function. Finally, all rotations were summed to obtain the scattered profile (Geyer \& Karastergiou 2016).

To determine the intrinsic parameters of the Crab pulsar profile, we fit scattered model profiles to the four observations with the lowest pulse scatter-broadening time scales. These observations were fully averaged in time and collapsed in frequency to 32 frequency channels of $2.5 \mathrm{MHz}$ in width. The parameters of the intrinsic profile of each channel were fitted simultaneously with the pulse scatter-broadening time scale $\tau_{\phi}$, and an offset in the baseline flux. The pulse scatterbroadening time scale in these four observations varied between 0.25 to $0.47 \mathrm{~ms}$ at a reference frequency of $350 \mathrm{MHz}$, with pulse scatter-broadening powerlaw indices of -3.8 to -4.2 . Flux uncertainties were estimated from the off-pulse region at pulse phases $0.0<\phi<0.1$ (with the MP at $\phi=0.25$ ). We find that the phase offset of the IP and the precursor with respect to the MP, as well as the widths of the three components, do not vary significantly with frequency over our available bandwidth. The relative amplitudes of the IP and precursor, measured with respect to the MP, do vary with frequency over our observed bandwidth, decreasing by 0.12 percent per $\mathrm{MHz}$. The fitted values are given in Table 1 and are compared to previously derived values. We expect some difference between our values and those derived at $610 \mathrm{MHz}$ as the intrinsic profile of the Crab is known to vary with frequency (e.g. Graham-Smith et al. 2011). Our values match well with those derived by Backer et al. (2000) at $350 \mathrm{MHz}$, apart from some difference between the precursor position and width. We use our intrinsic profile parameters to create a frequency dependent model for the intrinsic pulse profile of the Crab pulsar that is valid for our observing bandwidth

The overall shift of the profile with respect to frequency indicates that residual dispersion was still present. We were able to remove the residual dispersion by fitting a $v^{-2}$ dispersion dependence to each individual epoch. We conservatively estimate the error on our fitting to be $0.005 \mathrm{pc} \mathrm{cm}^{-3}$ $(0.07 \mathrm{~ms})$, which corresponds to less than two pulse phase bins and is significantly smaller than the scatter-broadening time scales, so it will not affect our measurements.

As some of our observations show the presence of a delayed echo of the intrinsic pulse profile, we expand our model by including additional scattering screens. The pulse scatter-broadening function is expanded to allow the inclusion of an arbitrary number $n$ of scattering screens through $s(t)=\sum_{i=0}^{n} \frac{1}{\tau_{i}} \exp \left(-\frac{t-t_{i}}{\tau_{i}}\right) H\left(t-t_{i}\right)$. Here, $\tau_{i}$ and $t_{i}$ are the pulse scatter-broadening time scale and the time offset of screen $i$, respectively. The time offsets are referenced to that of the first scattering screen, for which we define $t_{0}=0$. 


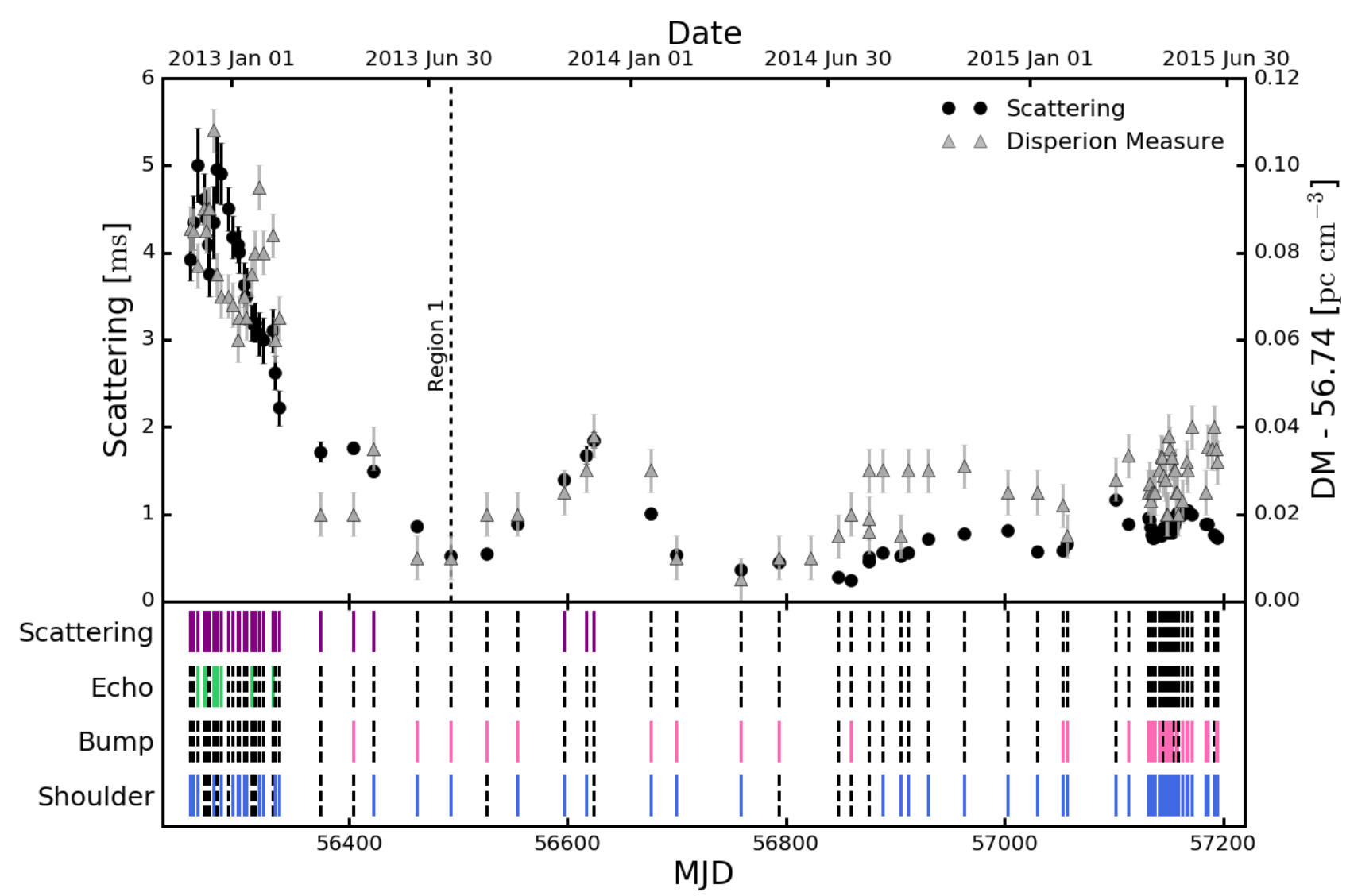

Figure 3. Properties of the Crab pulsar at $350 \mathrm{MHz}$ over time. The top panel shows the DM and pulse scatter-broadening, using a single-screen model. The bottom panel shows how often certain features occur. In the bottom panel a black dashed mark means that the feature is not present in that observation, a solid coloured mark means that the feature is present. Examples of these features are shown in Fig. 1 (with the same colour coding). Some features, particularly bumps and shoulders, may be hidden during periods of increased pulse scatter-broadening.

\section{RESULTS}

We modelled all of the observed profiles using models assuming one, and two scattering screens, some examples of models and residuals are shown in Fig. 4. For those profiles that have two clear peaks, such as in Fig. 4b, we find that a two screen model is effective and that the relative position of the second peak, or echo, to the first peak can be measured using a two screen model; however, there are only 4 observations with the echo as a clear, separate peak. As such, we cannot investigate the evolution of the separation between the echo and main profile in time.

In some cases we observe the echo to be brighter than the main profile. While the observation on 2012 December 15, see Fig. 1d, is the only profile with this feature clearly evident, there are five other profiles that suggest an echo brighter than the main profile. A possible explanation for this feature is plasma lensing, which is a refractive effect where an irregularity in a plasma screen acts as a lens. As the plasma has a negative refractive index this causes the light to diverge. Plasma lensing is observed as increases and decreases in flux density in a pattern of caustic spikes (Clegg et al. 1998) and can therefore explain a brighter echo compared to the original peaks.

Using the method described in Sec. 2.3 we attempted to fit profiles with shoulder features using models with two thin-screens. An example of the best fit to a profile with a shoulder feature is shown in Fig. 5. In contrast to modelling exponential echoes with two thin-screens the morphology of the shoulder is not well modelled by the thin-screen approximation, in particular the model cannot reproduce the smooth nature of the shoulder.

With higher cadence observations available in 2015 we are able to track bumps and shoulders in time. We find that over time the bumps approach the MP and IP, become shoulders, overlap the MP and IP, and later recede in a reverse manner; see Fig. 6. This indicates that shoulders and bumps are the same feature, where shoulders can be defined as bumps that are too close in phase to the MP and IP to be separated as individual features on the pulse profile. We do not observe shoulders or bumps evolving into echoes or vice versa. A similar effect to that seen in Fig. 6 was observed by Backer et al. (2000) and Lyne et al. (2001) in 1997, where the features they observed receded over 150 days after the initial approach. Lyne et al. (2001) observed quadratic paths of similar features approximately eight times in daily observations of the Crab pulsar from 1984 to 1998 and therefore suggest that they occur approximately every two years. However, although our cadence is not high enough to track 
Table 1. The parameters of the intrinsic profile from our model at $350 \mathrm{MHz}$ compared to the results of Backer et al. (2000) and Lyne et al. (2013). The amplitude values are relative to the MP, which has an amplitude set to 1.0. The position is the phase position in degrees and is measured relative to the MP position. The errors quoted here are $1 \sigma$ errors.

\begin{tabular}{lllrrr}
\hline & Frequency & Component & Amplitude & Position $\left(^{\circ}\right)$ & Width $\left(^{\circ}\right)$ \\
\hline Our results & \multirow{2}{*}{$350 \mathrm{MHz}$} & Main pulse & 1.0 & 0.0 & $2.85(11)$ \\
& & Interpulse & $0.623(2)$ & $145.74(4)$ & $3.36(10)$ \\
& & Precursor & $0.210(1)$ & $-18.25(11)$ & $13.9(2)$ \\
\hline Backer et al. (2000) & \multirow{2}{*}{$327 \mathrm{MHz}$} & Main pulse & $1.0(8)$ & $0.00(5)$ & $2.80(1)$ \\
& & Interpulse & 0.67 & 145.72 & $3.5(1)$ \\
& & Precursor & $0.29(5)$ & $-17.8(5)$ & $16.4(6)$ \\
& \multirow{2}{*}{$610 \mathrm{MHz}$} & Main pulse & $1.00(8)$ & $0.00(14)$ & $3.3(3)$ \\
& & Interpulse & 0.48 & 145.6 & $4.1(3)$ \\
& & Precursor & $0.06(2)$ & $-19.4(9)$ & $12(4)$ \\
\hline Lyne et al. (2013) & \multirow{2}{*}{$610 \mathrm{MHz}$} & Main pulse & 1.0 & 0.0 & \\
& & Interpulse & 0.565 & 145.59 & \\
& & Precursor & 0.197 & -18.42 & \\
\hline
\end{tabular}

the features, our data at $350 \mathrm{MHz}$ show bump and shoulder features consistently throughout the two and a half years of our observations. This highlights that both observing frequency and cadence are important considerations for future observations of transient profile features for the Crab pulsar.

To study the pulse scatter-broadening properties we use the frequency dependent intrinsic profile and single screen approximation to determine the residual dispersion measure as well as the pulse scatter-broadening time scale and pulse scatter-broadening-frequency powerlaw $\left(\tau \propto v^{\alpha}\right)$ dependence of all observations. Although using the single screen approximation is not optimal, it gives a first order estimate of the total extent of the pulse scatter-broadening of the pulse profile. Here, we again use those observations, which were fully a averaged in time and collapsed to 32 channels in frequency. We then model all 32 channels for each observation using single-screen models as described in Sec. 2.3 and find the average of the resulting pulse scatter-broadening time scales, $\tau$. The DM and resulting average pulse scatter-broadening values are shown in Fig. 3, highlighting the increased pulse scatter-broadening and DM during the period where echoes are observed on the pulse profile of the Crab. The powerlaw index of all observations is $\alpha=-3.9 \pm 0.5$. This result is consistent with the expected powerlaw index, $-4.4<\alpha<-4$, for thin screen scattering.

\section{DISCUSSION}

In our observations both exponential and non-exponential echoes vary in phase position (see Fig. 6 and Appendix A) and amplitude over time. We therefore discard the possibility that these features are intrinsic, i.e. related to other pulse profile features seen at different frequencies such as the low-frequency precursor, and instead assume that they are caused by effects in the ISM or Crab nebula. Previous analyses of past instances of additional pulse scatter-broadening (e.g. Backer et al. 2000; Graham-Smith et al. 2011) have assumed that the additional pulse scatter-broadening and echoes are caused by a second scattering screen. The second screen is generally assumed to be from a filament, sheet, or blob in the Crab nebula and the first screen is assumed to be the ISM. Further modelling and observations are required to fully investigate the mechanisms causing exponential echoes and non-exponential echoes, and whether both features arise from the nebula, or from the ISM, and whether they are both caused by the same mechanism. To facilitate further investigation, the data is available online ${ }^{4}$. We would define the "echoes" observed by Lyne \& Thorne (1975) as exponential echoes, and the "echoes" observed by Backer et al. (2000) as non-exponential echoes. Non-exponential echoes have been observed on a different pulsar, PSR B2217+47, by Michilli et al. (2018) and were attributed to propagation effects the ISM.

If we assume that echoes are caused by screens or filaments in the Crab nebula we can investigate the properties of this screen, such as its size and the scattering angle. We define the end of the screen to be the local minimum at 2013 April 22 which is indicated as region 1 in Fig. 3, with the start defined as the start of our data set. The end of this region was chosen because the pulse scatterbroadening reaches a minimum and we see no obvious echo feature in this observation. Choosing these limits means that the screen was crossing the line of sight to the pulsar for at least $\approx 139$ days.

Given our observing boundaries, and if we assume that the velocity of the filament relative to the Crab pulsar is due completely to the proper motion of the pulsar (GrahamSmith et al. 2011), $120 \mathrm{~km} \mathrm{~s}^{-1}$ (Kaplan et al. 2008), this filament is found to extend for at least 9.6 AU. The period of additional pulse scatter-broadening observed by GrahamSmith et al. (2011) had a shorter duration than the pulse scatter-broadening period discussed above. They estimated the filament causing the pulse scatter-broadening to extend for $\approx 10$ days corresponding to $0.72 \mathrm{AU}$.

If we assume that the second screen is within the Crab nebula then we can calculate the scattering angle. The scattering angle, $\theta$ in Fig. 7, is the angle between the direct line of sight to the pulsar $\left(D_{1}+D_{2}\right)$ and the direction to the screen producing the echo. If distance $D_{2} \gg D_{1}$, as is the case if the second screen is in the nebula, then we can as-

${ }^{4}$ https://doi.org/10.5281/zenodo. 1481083 

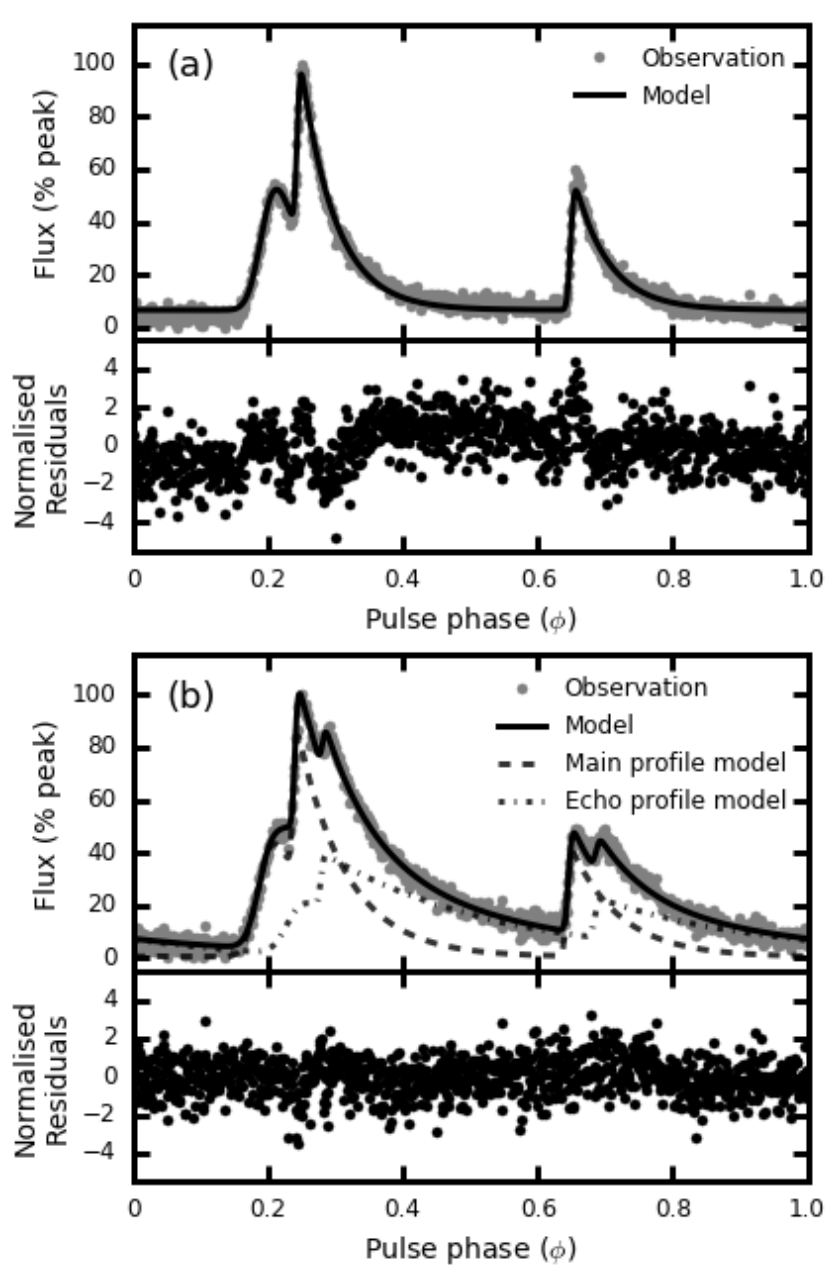

Figure 4. Examples of modelling echoes assuming (a) a single thin scattering screen and (b) two thin scattering screens. (a) and (b) show one $8 \mathrm{MHz}$ channel of the same observations as in Fig. $1 \mathrm{~b}$ and $\mathrm{c}$ respectively. The normalised residuals for the models are shown below each pulse profile, where the normalised residuals are the profile subtracted from the data and divided by the standard deviation of the off-pulse noise. The reduced $\chi^{2}$ values for the full fit to the pulse profile are 1.7 and 0.9 for (a) and (b) respectively.

sume that $D_{1}+D_{2} \approx D_{2}$. This means that $\theta$ can then be found using:

$\Delta t \approx \frac{D_{1} \theta^{2}}{2 c}$

where $\Delta t$ is the time delay between the arrival of the main profile and the echo, $D_{1}$ is the distance shown in Fig. 7, and $c$ is the speed of light.

At $350 \mathrm{MHz}$ on 2012 December 18 the shift in time between the main profile and the echo is $\Delta t \approx 1.5 \mathrm{~ms}$. The Crab pulsar is $2 \mathrm{kpc}$ from the Earth (Kaplan et al. 2008) and the nebula is an ellipsoid of $\approx 8.3 \times 10^{5} \mathrm{AU} \times 6.2 \times 10^{5} \mathrm{AU}$ (Green 2014). The termination shock radius of the cold pulsar wind is at $\approx 2.1 \times 10^{4} \mathrm{AU}$ from the pulsar (Lyne \& Thorne 1975 ). If we assume the screen is somewhere between the pulsar wind termination shock and the outer edge of the nebula we find that $\theta$ is between 3.52 arcsec and 0.56 arcsec. This means that $D_{3}$ would be between $2.2 \mathrm{AU}$ and $0.35 \mathrm{AU}$.

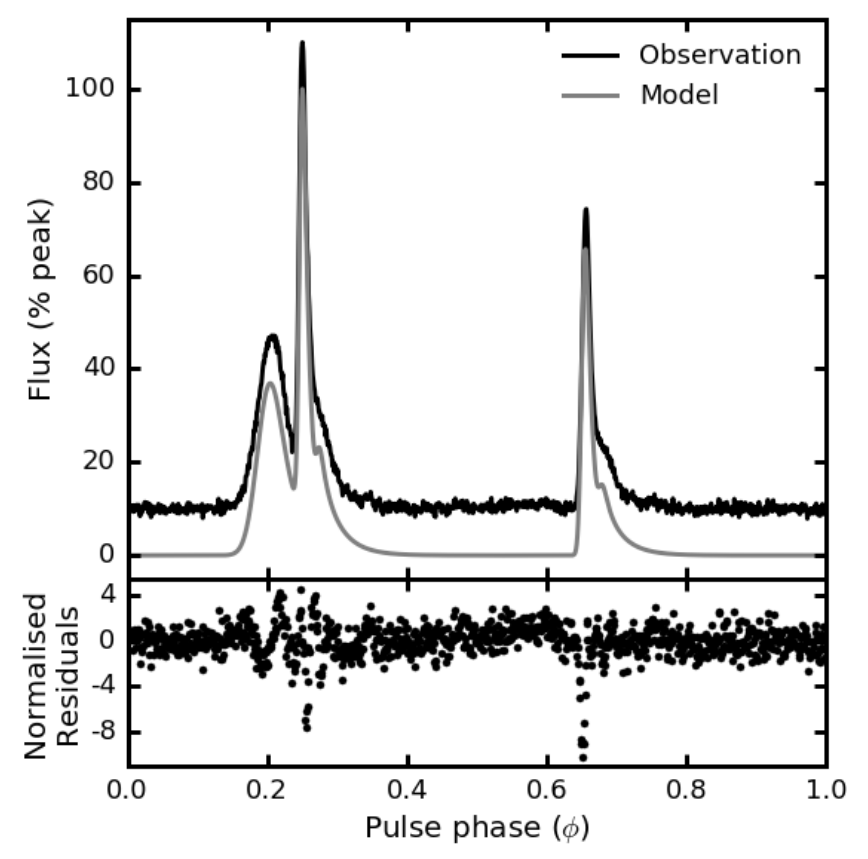

Figure 5. An example of a thin-screen approximation fit to a pulse profile with a shoulder feature. This is one $8 \mathrm{MHz}$ channel of the same observation as shown in Fig. 1e, 2014 May 16. The model fit to the data is a two-screen model, a screen for the main profile and a screen for the shoulder. This figure shows that the smooth nature of shoulder features cannot be reproduced using the thin-screen approximation. The residuals in the lower plot show the profile subtracted from the data and divided by the standard deviation of the off-pulse noise. The residuals show that this model also does not reproduce the precursor, MP or IP correctly. The reduced $\chi^{2}$ value of the full fit to the pulse profile is 2.2. The observation has been offset from the model by 10 flux units.

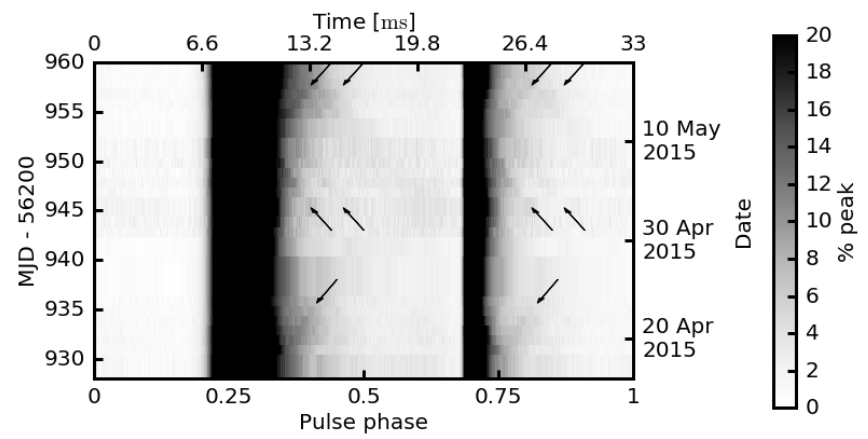

Figure 6. Observations of the Crab pulsar where bump features (i.e. non-exponential echoes) can be traced over a timescale of a couple of weeks. The bumps (indicated with arrows) approach and recede from the MP and IP over time between lines marked between 57145 and 57160 . An earlier bump can be seen to recede from the MP and IP from the start of the figure for about 10 days. The greyscale has been adjusted manually to make the bumps stand out more clearly. 


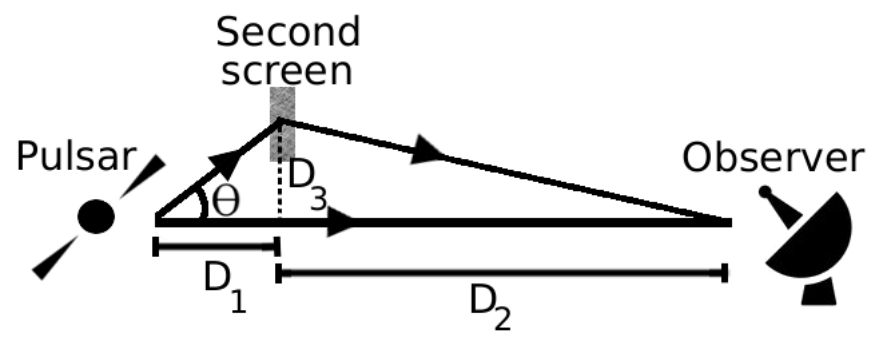

Figure 7. Diagram showing scattering angle and distance between the pulsar and the observer.

While we were able to use two-screen models using the thin-screen approximation to model observations with exponential echoes, we were unable to model non-exponential echoes using a thin screen model because they have a smooth Gaussian-like structure compared to exponential echoes. It is possible that the thin-screen assumption does not hold for these features, but further investigation and modelling are required to understand these features.

In our observations and the 1974 observations it is not clear whether exponential echoes evolve in time by first approaching the main profile, overlapping the main profile, then later receding, in a similar way to non-exponential echoes. We expect the evolution in time to be similar, assuming that both exponential and non-exponential echoes are caused by material crossing the line of sight to the pulsar. Higher cadence observations are required to investigate this, but due to the increased scattering when exponential echoes occur it may be difficult to track how they vary over time.

\section{CONCLUSIONS}

We observe pulse scatter-broadening, exponential echoes, and non-exponential echoes consistently in WSRT observations at $350 \mathrm{MHz}$ of the Crab pulsar over two and a half years. We find that we can model pulse scatter-broadening and exponential echoes with a thin-screen scattering approximation; however, this model cannot replicate non-exponential echoes. We observe that shoulders and bumps are the same non-exponential echoes, but at different phases relative to the main profile. Non-exponential echoes approach the MP and IP over time and later recede, similar to previous observations. As this is only the third time echoes have been observed on Crab pulse profiles, further investigation is required to determine whether exponential echoes and non-exponential echoes are produced by the same inhomogeneities in the ISM or Crab nebula and whether they are caused by the same scattering mechanism. We rule out intrinsic profile changes as exponential and non-exponential echoes vary in phase position and amplitude over time.

Our observations show that daily monitoring of the Crab pulsar at $\sim 350 \mathrm{MHz}$ would be a useful tool for further investigating transient features caused by propagation effects. It would be interesting to investigate these features using Crab pulsar giant pulses as giant pulses are intrinsically very bright and narrow. They also have only a single component, which would make the effects of pulse scatterbroadening more apparent and would make fitting easier, even when the pulse scatter-broadening time is much greater than the pulse period. Both higher cadence observations at $\sim 350 \mathrm{MHz}$ and investigations using giant pulses would lead to further insight on which material in the Crab nebula causes these effects, how often they occur, and their evolution in time.

\section{ACKNOWLEDGEMENTS}

The Westerbork Synthesis Radio Telescope is operated by the Netherlands Institute for Radio Astronomy (ASTRON) with support from The Netherlands Foundation for Scientific Research (NWO). LND and BWS acknowledge support from the European Research Council (ERC) under the European Union's Horizon 2020 research and innovation programme (grant agreement No 694745). We would like to thank Andrew Lyne, Marten van Kerkwijk, and James McKee for useful and interesting discussions. LND would like to thank the ASTRON/JIVE summer studentship programme for a fellowship in 2014. We thank the referee, assistant editor, and scientific editor for their constructive comments on the manuscript.

\section{REFERENCES}

Baars J. W. M., Hooghoudt B. G., 1974, A\&A, 31, 323

Backer D. C., Wong T., Valanju J., 2000, The Astrophysical Journal, 543, 740

Clegg A. W., Fey A. L., Lazio T. J. W., 1998, ApJ, 496, 253

Cordes J. M., 1986, ApJ, 311, 183

Geyer M., Karastergiou A., 2016, MNRAS, 462, 2587

Graham-Smith F., Lyne A., Jordan C., 2011, MNRAS, 410, 499

Green D. A., 2014, Bulletin of the Astronomical Society of India, 42,47

Hotan A. W., van Straten W., Manchester R. N., 2004, Publ. Astron. Soc. Australia, 21, 302

Hunter J. D., 2007, Computing In Science \& Engineering, 9, 90

Isaacman R., Rankin J. M., 1977, ApJ, 214, 214

Jones E., Oliphant T., Peterson P., et al., 2001-, SciPy: Open source scientific tools for Python, http://www.scipy.org/

Kaplan D. L., Chatterjee S., Gaensler B. M., Anderson J., 2008, ApJ, 677, 1201

Karuppusamy R., Stappers B., van Straten W., 2008, PASP, 120, 191

Karuppusamy R., Stappers B. W., van Straten W., 2010, Astronomy and Astrophysics, 515, A36

Karuppusamy R., Stappers B. W., Lee K. J., 2012, A\&A, 538, A7

Krishnakumar M. A., Joshi B. C., Manoharan P. K., 2017, ApJ, 846, 104

Kuzmin A. D., Kondrat'ev V. I., Kostyuk S. V., Losovsky B. Y., Popov M. V., Soglasnov V. A., D'Amico N., Montebugnoli S., 2002, Astronomy Letters, 28, 251

Kuzmin A., Losovsky B. Y., Jordan C. A., Smith F. G., 2008, Astronomy I\& Astrophysics, 483, 13

Lang K. R., 1971, ApJ, 164, 249

Lee L. C., Jokipii J. R., 1976, ApJ, 206, 735

Lewandowski W., Rożko K., Kijak J., Bhattacharyya B., Roy J., 2015, MNRAS, 454, 2517

Lyne A. G., Thorne D. J., 1975, Monthly Notices of the Royal Astronomical Society, 172, 97 
Lyne A. G., Pritchard R. S., Graham-Smith F., 2001, MNRAS, 321,67

Lyne A., Graham-Smith F., Weltevrede P., Jordan C., Stappers B., Bassa C., Kramer M., 2013, Science, 342, 598

McKinnon M. M., 2014, PASP, 126, 476

Michilli D., et al., 2018, MNRAS, 476, 2704

Moffett D. A., Hankins T. H., 1996, ApJ, 468, 779

Owen P. J., Barlow M. J., 2015, ApJ, 801, 141

Rankin J. M., Counselman III C. C., 1973, ApJ, 181, 875

Rankin J. M., Comella J. M., Craft Jr. H. D., Richards D. W., Campbell D. B., Counselman III C. C., 1970, ApJ, 162, 707 Rickett B. J., 1969, Nature, 221, 158

Staelin D. H., Reifenstein E. C., 1968, Science, 162, 1481

Williamson I. P., 1972, MNRAS, 157, 55

van Straten W., Bailes M., 2011, Publ. Astron. Soc. Australia, 28,1

\section{APPENDIX A: CRAB PULSE PROFILES}

Here we include the pulse profiles of the 84 WSRT observations of the Crab pulsar. All profiles are WSRT $350 \mathrm{MHz}$ observations of the Crab and have been averaged in time and collapsed in frequency. Every pulse profile has been rotated such that the maximum peak is at $\phi=0.25$, and scaled such that the peak value is 1.0 .

This paper has been typeset from a $\mathrm{T}_{\mathrm{E}} \mathrm{X} / \mathrm{LAT}_{\mathrm{E}} \mathrm{X}$ file prepared by the author. 

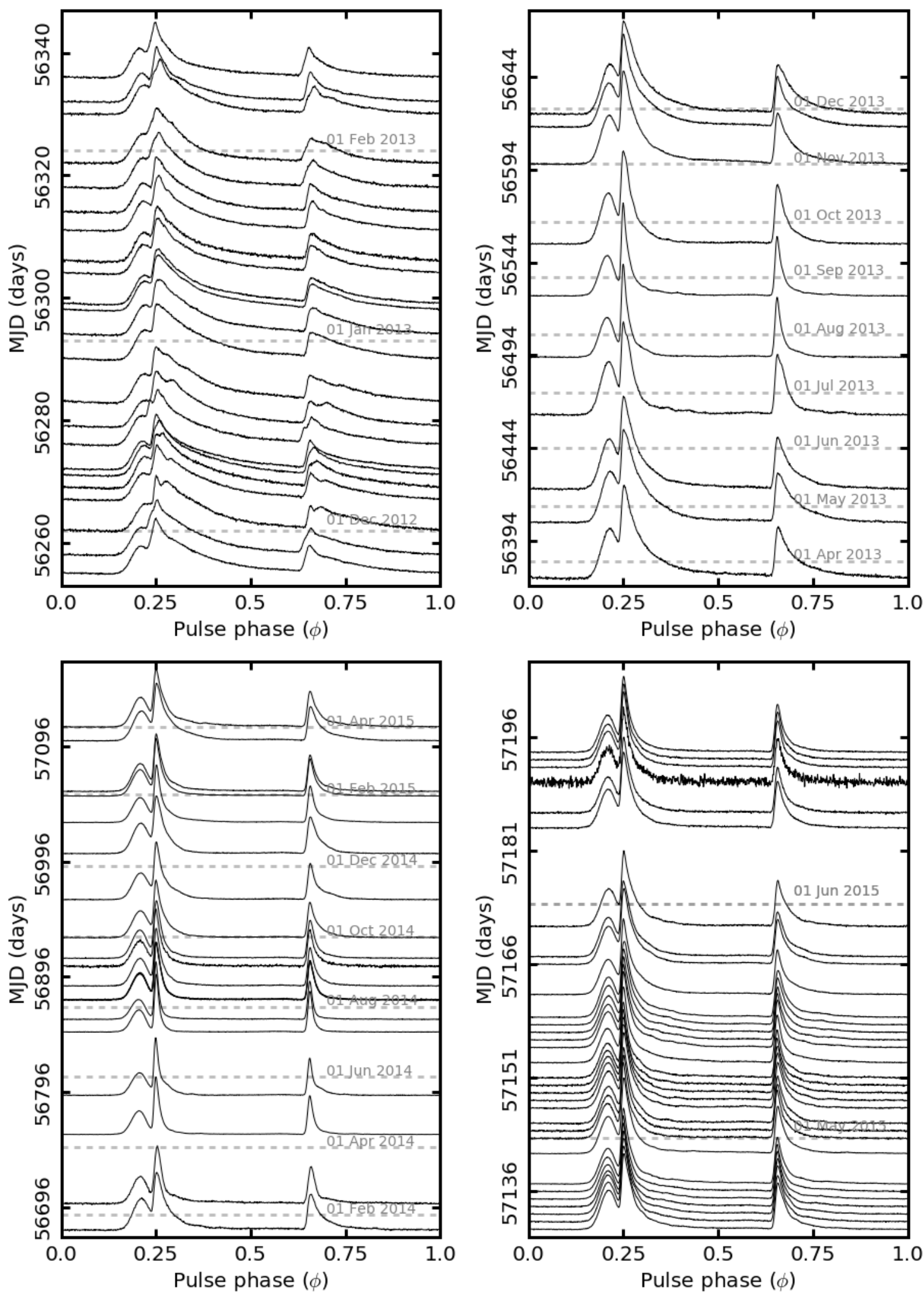

Figure A1. Pulse profiles of the Crab pulsar at $350 \mathrm{MHz}$ observed by WSRT from late 2012 until mid 2015 . Each line represents one observation, and each profile has been scaled such that the maximum is 1.0 and the minimum is 0.0 , and rotated such that the main peak is at $\phi=0.25$. 